\title{
Effects of Reallocating Physical Activity, Sedentary Behaviors, and Sleep on Mental Health: A Cross-Sectional Examination of Canadian Adolescents in the COMPASS Study
}

Jenna Gilchrist ( $\nabla$ jenna.gilchrist@uwaterloo.ca )

University of Waterloo https://orcid.org/0000-0002-7323-0953

\section{Kate Battista}

University of Waterloo School of Public Health and Health Systems

\section{Karen A. Patte}

Brock University

\section{Guy Faulkner}

The University of British Columbia

\section{Valerie Carson}

University of Alberta

\section{Scott T. Leatherdale}

University of Waterloo School of Public Health and Health Systems

\section{Research}

Keywords: isotemporal substitution modelling, MVPA, screen time, homework, depression, anxiety, flourishing

Posted Date: June 8th, 2020

DOI: https://doi.org/10.21203/rs.3.rs-33268/v1

License: (c) (i) This work is licensed under a Creative Commons Attribution 4.0 International License. Read Full License 


\section{Abstract}

Background: Physical activity, sedentary behavior, and sleep are associated with mental health in adolescents. Mental health may depend not only on the amount of time spent in a specific activity, but also on the activity it displaces. The aim of this study was to examine the impact of reallocating 15 minutes of time spent in one health behavior with 15 minutes in another on adolescent mental health.

Methods: Cross-sectional data from the students participating in the COMPASS Study (2018-2019) were analyzed ( $N=$ 46,413). Participants self-reported the amount of time they spent engaged in moderate-to-vigorous physical activity (MVPA), doing homework and using screens, and their sleep duration on average each day. Participants also selfreported depressive symptoms, anxiety, and flourishing. Data were analyzed using isotemporal substitution modeling.

Results: Non-linear associations were evident between sleep and the mental health outcomes, so analyses were stratified by sleep duration based on whether students reported meeting current guidelines or not. Among adolescents getting less than the recommended amount of sleep, replacing any behaviour with sleep was generally associated with better mental health outcomes. Conversely, among adolescents getting adequate sleep, the findings did not support replacing other behaviors with sleep with the exception of screen time. Replacing homework and MVPA with sleep was associated with less flourishing regardless of sleep duration. Of the two sedentary behaviors assessed, replacing screen time with homework was associated with less depression and greater flourishing. However, replacing any behavior with homework was associated with greater anxiety regardless of whether participants reported adequate sleep or not.

Conclusions: Replacing screen time with any behaviour may be better for mental health outcomes. Results provide further support for the critical role of sleep in promoting healthy development during adolescence, though more sleep than is recommended may confer little benefit for mental health. Youth getting adequate sleep may benefit from replacing screen time with homework or MVPA. The findings demonstrate that mental health benefits may be obtainable at intervals as short as 15 minutes.

\section{Background}

Mental health contributes to a large proportion of the global burden of disease. ${ }^{1}$ Anxiety and depression are among the most common mental disorders with increasing incidence during adolescence thus positioning this period as a critical time for intervention. ${ }^{2}$ Though mental illness has largely been the prevailing focus of research on mental health, current conceptions position mental health not simply as the absence of illness, but also the presence of well-being. ${ }^{3}$ Capturing both dimensions of mental illness (e.g., symptoms of depression and anxiety) and well-being (e.g., flourishing) is therefore needed to holistically assess mental health. An understanding of the modifiable behaviors that are associated with mental health in adolescents are needed such that targeted interventions can be developed to aid prevention and treatment.

A growing body of research supports the beneficial effects of moderate-to-vigorous physical activity (MVPA) on mental health among adolescents. ${ }^{4}$ Additionally, sleep plays a critical role in promoting optimal mental health. ${ }^{5}$ Researchers have noted a non-linear relationship between sleep and mental health status with adolescents reporting less than seven hours of sleep or greater than nine hours of sleep per night reporting worse mental health status. ${ }^{6}$

Sedentary behaviors have been identified as a risk factor for poor mental health. ${ }^{7}$ Sedentary behaviours are any waking behaviours with energy expenditure less than or equal to 1.5 metabolic equivalents while in a sitting, reclining, or lying posture. ${ }^{8}$ Recently, researchers have advocated for greater attention to the types of sedentary behaviors in the prediction of health outcomes, with evidence to suggest that time spent in some types exert greater risk than time spent in others ${ }^{9}$. 
Adolescents commonly engage in screen-based sedentary behaviors during leisure-time (e.g., watching television, playing video games). Greater screen time is a noted risk factor for increased anxiety and depressive symptomology and decreased well-being ${ }^{10,7}$ though researchers have noted non-linear associations between screen time and the mental health of adolescents whereby no screen use or excessive screen use is associated with poorer mental health relative to moderate use. ${ }^{11,12}$ Homework also contributes to the proportion of time adolescents spend sedentary though is likely characterized by greater mental activity than leisure-time screen based sedentary behaviors. ${ }^{13}$ However, few studies have investigated specific associations between time spent doing homework and mental health outcomes. Researchers investigating non-recreational screen time (i.e., computer use for homework) reported no associations between time spent doing homework and mental health, but positive associations were noted for educational outcomes and persistence. ${ }^{10,14}$ Investigations of specific sedentary behavior types could guide prevention efforts with greater precision.

Given the finite time available to spend in any given behavior, understanding how displacing time spent in one behavior with another is associated with mental health outcomes can provide valuable insight for developing prevention interventions. Mental health may depend not only on the amount of time spent in a specific activity, but also on the activity it displaces. For example, the seemingly adverse effects of sedentary behaviors can often be attributed (at least in part) to the loss of other health promoting activities, such as participation in physical activity or getting adequate sleep. ${ }^{15,16}$ As such, identifying where to reallocate time for optimal health could provide insight into tailoring messages of health behaviors and would help to guide policy on effective preventative strategies for time use among adolescents.

Traditionally, researchers have examined the effects of health behaviors on mental health in isolation, without consideration of how one behavior necessarily displaces another behavior. Drawing from research in nutritional epidemiology, an isotemporal substitution paradigm has been developed as an analytic model to study the time substitution effects of one activity for another. ${ }^{17} \mathrm{~A}$ recent study investigating reallocating time between sleep, sedentary behavior, and physical activity generally found beneficial effects of replacing time spent sedentary or sleeping with physical activity for reducing mortality risk among middle aged and older adults. ${ }^{15}$ Interestingly, differences were noted among participants that reported sleeping less than 7 hours/night and those sleeping $>7$ hours/night. Among those sleeping $<7$ hours/night, replacing time spent sedentary with sleeping was associated with reduced mortality risk but an increased risk was noted among those sleeping $>7$ hours/night. The results of a recent systematic scoping review indicated that reallocating time spent sedentary to either light physical activity or MVPA was associated with a number of beneficial health outcomes in adults. ${ }^{18}$ However, the results of the scoping review highlight a dearth of studies among other populations including adolescents and further investigations into other outcomes beyond physical health, including mental health, is warranted. Further, a major limitation of most studies is the exclusion of sleep in many of the models. This is particularly salient in adolescent populations given the importance of sleep for healthy development. ${ }^{19}$

Of the extant research that has investigated mental health outcomes, the evidence generally supports reallocating time spent sedentary to MVPA. ${ }^{20,21}$ Differences between types of sedentary behavior on mental health have also been noted. Hallgren et al. (2019) explored the differential impact of mentally passive (e.g., television viewing, listening to music) versus active (e.g., office work, knitting) sedentary behaviour on risk of major depressive disorder among Swedish adults. ${ }^{22}$ Beneficial effects for mental health were found when replacing passive sedentary time with mentally active sedentary time.

No studies, to our knowledge, have examined how replacing specific health behaviors with one another is associated with mental health outcomes in adolescents. Therefore, the purpose of this study was to estimate the associations of alternating time spent in MVPA, passive sedentary behavior (i.e., screen time), mentally active sedentary behavior (i.e., homework), and sleeping with mental health outcomes among adolescents. Based on the extant literature, it was hypothesized that replacing other behaviors with sleep will be beneficial among those not getting adequate sleep but not 
for those getting adequate sleep. Replacing sedentary behaviors with MVPA was hypothesized to be associated with more favorable mental health outcomes among adolescents getting adequate sleep. Finally, it was hypothesized that replacing passive sedentary behaviors (i.e., screen time) with mentally active sedentary behaviors (i.e., homework) will be associated with better mental health outcomes.

\section{Method}

\section{Procedure}

This study used a sample of adolescents participating in the COMPASS (Cannabis, Obesity, Mental health, Physical activity, Alcohol, Smoking, Sedentary Behaviour) study. COMPASS is a prospective cohort study (2012-2021) that collects data from full school samples of students in grades 9 through 12 attending participating secondary schools across Canada. A full description of the COMPASS host study methods is available in print ${ }^{23}$ or online (www.compass.uwaterloo.ca). Participants were recruited using an active-information passive-consent procedure. Students provided self-reported responses to questions pertaining to health behaviors and mental health during class time. All procedures were approved by the University of Waterloo Office of Research Ethics (ORE 30118) and appropriate school board committees.

\section{Participants}

This study used cross-sectional student-level data from Year 7 (2018-2019) of the COMPASS cohort. The total sample was comprised of 74, 501 students from 136 schools. Participants in Quebec in grades Secondaire 1 and Secondaire 2 at the time of data collection were removed from the analysis leaving a total sample of 60,610 Canadian students in grades 9-12 from 134 schools in Quebec (50 schools), Ontario (61 schools), Alberta (8 schools) and British Columbia (15 schools). The participation rate for Year 7 was $84.2 \%$. Missing respondents were primarily a result of scheduled spares or absenteeism during data collection.

\section{Measures}

\section{Health Behaviors}

Screen Time. Participants were asked to indicate the amount of time they usually spend each day on the following activities: i) watching/streaming TV shows or movies, ii) Playing video/computer games, iii) surfing the Internet, and iv) texting/messaging/emailing. Response options for each type of sedentary behavior included separate estimates for hours $(0-9)$ and minutes $(0,15,30,45)$. Scores were added across each modality to represent the total screen time participants engaged in per day, on average.

Homework. Time spent on homework was assessed with a single item asking participants to indicate the amount of time spent on homework each day, on average. The response options followed the same format as the screen time items.

Sleep. Sleep duration was assessed using the same scale as the sedentary behaviors. Participants indicated how much time they usually spent sleeping each day with response options ranging from 0-9 hours and the number of minutes in 15-minute intervals.

Physical Activity. Time spent in MVPA was assessed using two items modified from the School Health Action, Planning and Evaluation System (SHAPES) physical activity questionnaire which was designed for multiple large-scale schoolbased data collections. ${ }^{24}$ Participants were asked to indicate the amount of time spent in moderate and vigorous 
physical activity each day over the past week. Response options included hours (0-4) and minutes in 15-minute increments $(0$ - 45). Participants were provided with written examples of moderate activities (e.g., walking, biking to school, and recreational swimming) and vigorous activities (jogging, team sports, fast dancing, jump-rope) prior to providing their response. Time spent in each intensity was combined to reflect total time spent in MVPA and was averaged across the 7 days to reflect average time spent in MVPA on a given day in the past week. The validity of the scores from the items are consistent with other self-reported assessments of physical activity among adolescents. ${ }^{25}$

\section{Mental Health}

Depression. The 10-item Center for Epidemiologic Studies Depression Scale Revised-10 (CESD-R-10) ${ }^{26}$ was used to assess depressive symptoms. Participants were asked to indicate the number of days over the past week they experienced a number of symptoms (e.g., "I was bothered by things that usually don't bother me"). Response options ranged from 1 (None or less than 1 day) to 4 (5-7 days). Scale values were mean-imputed for students missing one or two responses. Internal consistency of the scores was $a=0.82$.

Anxiety. Anxiety symptoms were assessed using the Generalised Anxiety Disorder 7-item Scale (GAD-7). ${ }^{27}$ Participants indicated how often they had been bothered by problems such as difficulty controlling feelings of worry, trouble relaxing, nervousness, restlessness and irritability over the previous 2 weeks. Scale values were mean-imputed for students missing one or two responses. The internal consistency of the scores was $a=0.90$.

Flourishing. The Flourishing Scale ${ }^{28}$ consists of 8 -items designed to assess positive human functioning. Items assess dimensions of psychological well-being including positive relationships and feeling competent and meaningful in life (sample item: "I lead a purposeful and meaningful life"). The response scale was modified from the original 7-point Likert-scale to a 5-point Likert scale ranging from 1 (strongly agree) to 5 (strongly disagree). A single score was derived by summing participant responses across the 8-items. For ease of interpretation, the overall score was reversed such that higher scores reflect greater flourishing. Support for the construct validity of test scores and estimates of score reliability (coefficient $a)^{29}$ have been reported in the literature. ${ }^{28,30}$ Scale values were mean-imputed for students missing one or two responses. The internal consistency of the scores was $a=0.90$.

\section{Covariates}

Covariates measured included sex (female, male), grade (9-12), province, self-described ethnicity (White, Black, Asian, Latin American/Hispanic, Other/Mixed), weekly spending money as a proxy for socioeconomic status (SES), and selfreported height and weight to calculate weight status using age- and sex-adjusted cut points (recoded as underweight, normal-weight, overweight, obese, missing). ${ }^{31}$

\section{Data Analysis}

The distributions of the physical activity, screen time, homework, and sleep scores were examined. Based on the observed distributions of responses, students with missing data as well as unrealistic or outlier responses were removed. Sample characteristics were calculated and linear mixed models with random intercept were used to examine the impact of changes in MVPA, sleep, homework, and screen time on anxiety, depression and flourishing scores. All models controlled for sex, province, grade, ethnicity, weekly spending money, and BMI classification. Models with anxiety and flourishing outcomes controlled for depressive symptoms, while models with depressive symptoms controlled for anxiety. Random intercepts for each school were used to account for intraclass correlation (i.e. that students from the same school are more alike than students from different schools). All models were run using the GLMMIX procedure in SAS 9.4. 
Consistent with previous methods, ${ }^{32}$ isotemporal substitution models were used to examine the impact of replacing 15 minutes of either screen time, homework, MVPA or sleep with another activity. In isotemporal substitution models, total activity time is held constant and a series of models are run removing one activity at a time. The covariates for the remaining activities represent the effect of replacing the removed activity with an equivalent amount of time of the remaining activity. For example, to examine the impact of replacing 15 minutes of screen time with either MVPA or sleep, the isotemporal substitution model is expressed as:

The coefficient for total time represents the removed activity (i.e. screen time), while the coefficient represents the impact of replacing 15 minutes of screen time with 15 minutes of MVPA, the coefficient represents the impact of replacing 15 minutes of screen time with 15 minutes of sleep, and the coefficient represents the impact of replacing 15 minutes of screen time with 15 minutes of homework.

Partition models were also run to examine the impact of increasing MVPA, screen time, homework, or sleep, while holding all other activity types constant. Partition models represent the effect of adding an activity, as opposed to substituting it with another activity. Unlike isotemporal models, total time is not held constant.

Based on previous research, non-linear associations between sleep, homework, screen time, and MVPA and each outcome variable were examined. Quadratic and square-root terms were tested using random intercept models. Because isotemporal models require linear associations, when single or partition models showed evidence of a non-linear relationship, the isotemporal models were stratified so that associations could be examined separately.

\section{Results}

Students with missing values on mental health scales $(n=3,679)$ and covariates $(n=2,042)$ were removed. Using outlier criteria of three standard deviations from the mean, students who indicated average daily MVPA $(n=293)$ or homework time $(n=174)$ greater than 6 hours or sleep time $(n=2,969)$ less than 3 hours were removed, as were students who indicated a total activity time (including physical activity, screen time, homework, and sleep) of more than 24 hours ( $n=$ $5,040)$, resulting in a final analytic sample of 46,413 students. Sample descriptives are presented in Table 1. No meaningful non-linear associations were observed for sedentary behavior, homework, or MVPA. A significant quadratic relationship was observed for sleep and each mental health outcome. Based on the non-linear associations evident between sleep and the mental health outcomes, we further stratified our analyses by examining the associations at varying levels of sleep duration (i.e., $<8$ hours, ${ }^{3} 8$ hours) based on current guidelines. ${ }^{33}$

\section{Partition Models}

Results of the partition models are presented in Table 2. Among adolescents not meeting current sleep guidelines, screen time positively predicted anxiety (estimate $=0.01,95 \% \mathrm{Cl}=0.01,0.02$ ) and depression (estimate $=0.01,95 \% \mathrm{Cl}=0.01$, 0.02 ) and was negatively associated with flourishing (estimate $=-0.01,-0.02,-0.01$ ). Time spent doing homework was positively associated with anxiety (estimate $=0.07,95 \% \mathrm{Cl}=0.06,0.08$ ) and flourishing (estimate $=0.12,95 \% \mathrm{Cl}=0.11$, 0.13 ), and negatively associated with depression (estimate $=-0.06,-0.07,-0.05$ ). MVPA was positively associated with anxiety (estimate $=0.02,95 \% \mathrm{Cl}=0.01,0.03$ ) and flourishing (estimate $=0.15,95 \% \mathrm{Cl}=0.13,0.16$ ) but negatively associated with depression (estimate $=-0.03,95 \% \mathrm{Cl}=-0.04,-0.01$ ). Finally, sleep was negatively related to both anxiety (estimate $=-0.02,95 \% \mathrm{Cl}=-0.03,-0.01$ ) and depression (estimate $=-0.15,95 \% \mathrm{Cl}=-0.16,-0.14$ ) and positively related to flourishing (estimate $=0.07,95 \% \mathrm{Cl}=0.06,0.08$ ).

Among those meeting current sleep guidelines, partition models were similar for both screen time and homework, however differences emerged for MVPA and sleep. MVPA was not associated with either anxiety or depression but was positively associated with flourishing. Sleep was not significantly associated with anxiety, depression, or flourishing. 


\section{Isotemporal Substitution Model: Relationships Across Sleep Duration Categories}

The effects of replacing sedentary behaviors, sleep, and MVPA with the mental health outcomes stratified across sleep duration categories are presented in Table 2.

Replacing sedentary behaviors and MVPA with sleep. In general, better mental health outcomes were noted when screen time, homework, or MVPA was replaced with sleep among those sleeping less than eight hours per night. Of interest, however, replacing homework and MVPA with sleep was negatively associated with flourishing. While some similarities were evident among those meeting guidelines, there were noted differences. For example, although the benefits of replacing screen time were evident across both groups, replacing screen time with sleep was not associated with anxiety among those meeting current sleep guidelines. Further, among those meeting guidelines, replacing MVPA with sleep was not associated with anxiety or depression and similar to the other group was negatively associated with flourishing. As well, replacing homework with sleep was positively associated with depression, and similar to those not meeting guidelines, was associated with less flourishing.

Replacing sedentary behaviors with MVPA. Similarly, better mental health outcomes were generally noted when either sedentary behavior (i.e., screen time or homework) was replaced with MVPA. A notable exception to this was replacing homework with MVPA when depressive symptoms were the criterion variable of interest. Across both groups, replacing 15 minutes of homework with 15 minutes of MVPA was associated with greater depressive symptoms. Associations were of similar magnitude across groups. The only difference that emerged between groups pertained to replacing screen time with MVPA for anxiety. Among those reporting less than 8 hours of sleep a night, replacing screen time with MVPA was not associated with anxiety whereas this association was negative among those meeting current guidelines.

Replacing screen time with homework. When 15 minutes of screen time was replaced by 15 minutes of homework, participants reported greater anxiety, less depression, and greater flourishing, regardless of sleep duration.

\section{Discussion}

This study investigated how displacing 15 minutes of MVPA, sedentary behavior, or sleep with equivalent time in another behavior was associated with mental health outcomes in adolescents using isotemporal substitution modelling. There was evidence of non-linear associations between sleep and mental health, consistent with previous research. ${ }^{6}$ Consequently, all findings were stratified across sleep duration. While similarities emerged between those meeting sleep guidelines and those not when sleep replaced other behaviors, differences were evident as well. Replacing screen time with sleep was beneficial for both groups, however magnitudes were greater for those not meeting sleep guidelines. Among participants not meeting sleep guidelines, replacing 15 minutes of MVPA or homework with 15 minutes of sleep was associated with less anxiety and depression. These results contrast findings that increased engagement in MVPA mitigates the detrimental effects of insufficient sleep, though past research has not been focused on mental health. ${ }^{19,34}$ Conversely, among adolescents meeting current sleep guidelines, there was little benefit of replacing MVPA or homework with sleep. These findings are in line with our hypotheses and support previous research demonstrating the non-linear relationship between sleep and mental health whereby additional sleep beyond recommended guidelines is negatively associated with mental health or confers no additional benefits. ${ }^{6}$ However, the equivalent findings between groups when sleep replaced screen time suggests that any differences may depend on the behavior that sleep displaces. Further, despite previously reported positive associations between MVPA and mental health, ${ }^{35}$ the beneficial outcomes of MVPA may be predicated on adolescents getting adequate sleep.

In contrast to the findings for anxiety and depressive symptoms, replacing homework and MVPA with sleep was negatively associated with flourishing regardless of sleep duration. These findings highlight the need to examine both 
dimensions of mental health (i.e., ill-being and well-being) given the differing associations noted. The negative associations with flourishing may be explained in light of research on eudaimonic pursuits and their subsequent effects on well-being. ${ }^{36}$ It is possible that activities like homework and MVPA that are characterized by engagement, mastery, and a sense of agency and personal growth promote flourishing while displacing time in these activities detracts from flourishing. ${ }^{37,38}$ Further supporting this supposition, replacing screen time with the other behaviors was associated with greater flourishing where screen time aligns more closely with hedonic motives and is less likely to be considered a eudaimonic pursuit that would promote flourishing. ${ }^{39}$ This finding provides further evidence that the behaviors that comprise an individual's day contribute differently to dimensions of mental health with some behaviors contributing more to well-being and others to ill-being.

Although limiting the amount of time spent sedentary or interacting with screens is recommended, ${ }^{33}$ there is little research to help guide which behaviors should displace time spent sedentary and the relative effects of replacing different types of sedentary behavior on adolescents' mental health. Within adult samples, replacing time spent sedentary with MVPA is generally associated with better health outcomes. ${ }^{18}$ The results of our study indicate that replacing screen time with MVPA was consistently associated with better mental health, particularly greater flourishing, while replacing homework with MVPA was associated with less anxiety and greater flourishing but increased depressive symptoms. However, it is important to note that the effect sizes were small. Our findings add to the growing body of literature demonstrating the beneficial outcomes of replacing sedentary behavior with MVPA but illustrate that such effects may depend on the behavior it displaces. ${ }^{18,20,22}$

We also examined how replacing two types of sedentary behavior with one another was associated with mental health. Replacing screen time with homework was associated with greater anxiety, less depression, and greater flourishing regardless of sleep duration. These findings are consistent with Hallgren and colleagues ${ }^{22}$ who found replacing passive sedentary behavior with active sedentary behavior reduced the odds of depressive symptoms. Potential explanations as to why replacing passive sedentary behaviors exerts better health outcomes relative to mentally active sedentary behaviors have been put forth recently. ${ }^{9}$ Similar to the findings noted above, homework likely constitutes a eudaimonic pursuit relative to screen time which may also explain the beneficial mental health outcomes evidenced when homework displaced screen time. Taken together, these results provide useful direction for interventions and messaging targeting sedentary behaviors among adolescents and support recent suppositions and findings that not all sedentary behaviors have equivalent effects on mental health. ${ }^{14,18,21,23}$

Replacing any behavior with homework was consistently associated with greater anxiety. This speaks to the anxiety students may feel around homework or academic pursuits outside of school. ${ }^{40}$ However, research examining associations between homework and mental health are scarce and further research on this topic is warranted. While it seems likely that homework may be anxiety inducing itself, alternative explanations are also possible. The seemingly adverse effects of homework on anxiety may be attributable to loss of other behaviors important for mental health including adequate sleep. Time spent studying or doing homework is negatively related to time spent sleeping. ${ }^{41,42}$ Subsequently, limited sleep interferes with adolescents' ability to regulate emotions and is associated with increased anxiety. ${ }^{43,44}$ Alternatively, homework may lead to increased arousal and anxiety that interferes with sleep. ${ }^{45}$ Furthermore, the quality of motivation underpinning these behaviors may affect mental health. ${ }^{46}$ Relative to screen time or MVPA, which are largely pursued during leisure time, homework is likely to be perceived as less autonomous.

Implications of these findings are relevant at both the individual and policy level. At the individual level, there is opportunity for intervention during the bedtime hours. Screen-based technologies are pervasive among adolescents with estimates indicating almost all adolescents have at least one screen-based device in the bedroom. ${ }^{47}$ Exposure to screens during bedtime hours is negatively associated with sleep duration and quality. ${ }^{47}$ Replacing screen use with an earlier 
bedtime is recommended regardless of usual sleep duration. At the policy level, delayed school start times have shown initial promise for increasing sleep duration with delays in school start times as short as 10 minutes resulting in increased sleep duration. ${ }^{48,49}$ Researchers have also demonstrated that delayed school start times do not necessarily offset engagement in physical activity. Given the relatively short timeframe used when replacing behaviors (i.e., 15 mins), there exists opportunity for integrating short bouts of MVPA (e.g., activity breaks) either as a result of delayed school start times or dispersed throughout the day. Finally, based on our results and previous findings, students should be encouraged to replace time spent in behaviors that bring pleasure solely (i.e., hedonic pursuits) with behaviors that provide meaning and purpose (i.e., eudaimonic pursuits).

There are a number of strengths of the current study, including the use of a large, national dataset of adolescents. The relatively short duration of time use examined is likely to be a small enough increment of time that can be flexibly applied throughout the day. We accounted for sleep in our models, which is often excluded in models predicting mental health from physical activity, and a noted limitation of many isotemporal models. ${ }^{18}$ With few exceptions, the majority of research examining the effects of replacing one behavior with another has been conducted with adult and children samples while little research has examined these effects in adolescent samples. Further, despite clear evidence documenting associations between MVPA, sedentary behavior, and sleep on mental health outcomes, much of this research has focused on physical health and body composition with little research on understanding how displacing one behavior with another is associated with mental health outcomes. Finally, despite current conceptions of mental health as both the absence of ill-being and the presence of well-being, much of the extant research has focused on ill-being (i.e., depression, anxiety). ${ }^{3}$ Our study presents a more holistic understanding of the effects of activity displacement on adolescent mental health.

This study is not without limitations. All behaviors were self-reported which can result in biased estimates. ${ }^{50,51}$ Social media use was not directly assessed. Given the ubiquity of social media use among adolescents, screen time is likely underestimated. ${ }^{52}$ There may also have been overlap between the sedentary behavior domains or other behaviors if participants were completing homework on the computer, for example, or were being active while using screens (e.g., active video games). ${ }^{14,53}$ As well, the extent to which sedentary behaviors were characterized as mentally active or passive was not directly assessed. It is possible some forms of screen time may be more mentally active or passive relative to others. ${ }^{10,14}$ Assessing the amount of time individuals spent in front of screens relative to the content of screens is only one way of assessing the effects of screen-based behaviors and has some limitations particularly when mental health is the outcome of interest. ${ }^{54}$ The COMPASS survey restricts any reports greater than 9 hours and 45 minutse for sleep duration which may limit the inferences that can be made for those getting additional sleep. Neither light physical activity nor the modality or contextual factors associated with physical activity were included, both of which have implications for mental health. ${ }^{55}$ Light activity is likely one movement behaviour that significant time is allocated to each day. Finally, this study was cross-sectional so the direction of effects cannot be discerned.

\section{Conclusion}

Youth mental health has been regarded as an important priority for health care in Canada. ${ }^{56}$ There is a wealth of support for increasing MVPA, reducing sedentary time, and getting adequate sleep to promote positive mental health outcomes

among adolescents. ${ }^{6,57,35}$ However, due to the finite time available to engage in these behaviors and engaging in one behaviour necessarily means displacing time spent in another, the effects of replacing one health behavior with another on mental health is of interest. Taken together, our findings indicate that among adolescents not meeting current sleep guidelines, sleep should be prioritized. Among adolescents meeting guidelines however, additional sleep is generally recommended except when replacing screen time. These findings provide further support for the critical role of sleep in promoting healthy development during adolescence. Differences were noted across sedentary behavior types and 
mental health dimensions. Of considerable importance, our findings demonstrate that mental health benefits may be obtainable by reallocating movement behaviours at intervals as short as 15 minutes.

\section{Declarations}

\section{Ethics approval and consent to participate}

This study was approved by the University of Waterloo Office of Research Ethics (ORE 30118) and appropriate school board committees. All students attending participating schools were invited to participate using active-information passive-consent parental permission protocols. Students could withdraw from the study at anytime.

\section{Consent for publication}

Not applicable.

\section{Competing interests}

The authors declare that they have no competing interests.

\section{Availability of data and materials}

COMPASS study data is available upon request through completion and approval of an online form: https://uwaterloo.ca/compass-system/information-researchers/data-usage-application. The datasets used during the current study are available from the corresponding author on reasonable request.

\section{Funding}

This research was supported by the Social Sciences and Humanities Research Council of Canada through a postdoctoral fellowship awarded to JG. The COMPASS study has been supported by a bridge grant from the CIHR Institute of Nutrition, Metabolism and Diabetes (INMD) through the "Obesity - Interventions to Prevent or Treat" priority funding awards (OOP-110788; awarded to SL), an operating grant from the CIHR Institute of Population and Public Health (IPPH) (MOP-114875; awarded to SL), a CIHR project grant (PJT-148562; awarded to SL), a ClHR bridge grant (PJT-149092; awarded to KP/SL), a CIHR project grant (PJT-159693; awarded to KP), and by a research funding arrangement with Health Canada (\#1617-HQ-000012; contract awarded to SL).

\section{Authors' contributions}

JDG conceptualized the paper and led the writing of the manuscript. KB conducted the statistical analysis. STL leads the COMPASS host study, and KAP leads the COMPASS Mental Health pilot study. All authors reviewed and provided feedback on drafts and approved the final manuscript.

\section{Acknowledgements}

The authors would like to thank the schools, school boards, and students that have participated in the COMPASS study, and all COMPASS team members and staff.

\section{References}

1. Vigo D, Thornicroft G, Atun R. Estimating the true global burden of mental illness. Lancet Psychiatry. 2016;3:171178. doi:https://doi.org/10.1016/S2215-0366(15)00505-2 
2. Saxena S, Funk M, Chisholm D. World Health Assembly adopts Comprehensive Mental Health Action Plan 20132020. The Lancet. 2013;381:1970-1971. doi:10.1016/S0140-6736(13)61139-3

3. Keyes CLM. The mental health continuum: From languishing to flourishing in life. J Health Soc Behav. 2002;43(2):207-222. doi:10.2307/3090197

4. Biddle SJH, Ciaccioni S, Thomas G, Vergeer I. Physical activity and mental health in children and adolescents: An updated review of reviews and an analysis of causality. Psychol Sport Exerc. 2019;42:146-155. doi:10.1016/j.psychsport.2018.08.011

5. Shochat T, Cohen-Zion M, Tzischinsky O. Functional consequences of inadequate sleep in adolescents: A systematic review. Sleep Med Rev. 2014;18(1):75-87. doi:10.1016/j.smrv.2013.03.005

6. Zhang J, Paksarian D, Lamers F, Hickie IB, He J, Merikangas KR. Sleep patterns and mental health correlates in US adolescents. J Pediatr. 2017;182:137-143. doi:10.1016/j.jpeds.2016.11.007

7. Gunnell KE, Flament MF, Buchholz A, et al. Examining the bidirectional relationship between physical activity, screen time, and symptoms of anxiety and depression over time during adolescence. Prev Med. 2016;88:147-152. doi:10.1016/j.ypmed.2016.04.002

8. Tremblay MS, Aubert S, Barnes JD, et al. Sedentary Behavior Research Network (SBRN) - Terminology Consensus Project process and outcome. Int J Behav Nutr Phys Act. 2017;14(1):75. doi:10.1186/s12966-017-0525-8

9. Hallgren M, Dunstan DW, Owen N. Passive versus mentally active sedentary behaviors and depression. Exerc Sport Sci Rev. 2020;48(1):20-27. doi:10.1249/JES.0000000000000211

10. Babic MJ, Smith JJ, Morgan PJ, Eather N, Plotnikoff RC, Lubans DR. Longitudinal associations between changes in screen-time and mental health outcomes in adolescents. Ment Health Phys Act. 2017;12:124-131. doi:10.1016/j.mhpa.2017.04.001

11. Ferguson CJ. Everything in moderation: Moderate use of screens unassociated with child behavior problems. Psychiatr Q. 2017;88(4):797-805. doi:10.1007/s11126-016-9486-3

12. Przybylski AK, Weinstein N. A large-scale test of the Goldilocks hypothesis: Quantifying the relations between digitalscreen use and the mental well-being of adolescents. Psychol Sci. 2017;28(2):204-215. doi:10.1177/0956797616678438

13. Arundell L, Fletcher E, Salmon J, Veitch J, Hinkley T. A systematic review of the prevalence of sedentary behavior during the after-school period among children aged 5-18 years. Int J Behav Nutr Phys Act. 2016;13(1):93. doi:10.1186/s12966-016-0419-1

14. Sanders T, Parker PD, del Pozo-Cruz B, Noetel M, Lonsdale C. Type of screen time moderates effects on outcomes in 4013 children: Evidence from the Longitudinal Study of Australian Children. Int J Behav Nutr Phys Act. 2019;16(1):117. doi:10.1186/s12966-019-0881-7

15. Stamatakis E, Rogers K, Ding D, et al. All-cause mortality effects of replacing sedentary time with physical activity and sleeping using an isotemporal substitution model: A prospective study of 201,129 mid-aged and older adults. Int J Behav Nutr Phys Act. 2015;12(1):121. doi:10.1186/s12966-015-0280-7

16. Stiglic N, Viner RM. Effects of screentime on the health and well-being of children and adolescents: A systematic review of reviews. BMJ Open. 2019;9(1):e023191. doi:10.1136/bmjopen-2018-023191

17. Willett WC, Howe GC, Kushi LH. Adjustment for total energy intake in epidemiologic studies. Am J Clin Nutr. 1997;65:1220-1228. doi:https://doi.org/10.1093/ajcn/65.4.1220S

18. Grgic J, Dumuid D, Bengoechea EG, et al. Health outcomes associated with reallocations of time between sleep, sedentary behaviour, and physical activity: A systematic scoping review of isotemporal substitution studies. Int $J$ Behav Nutr Phys Act. 2018;15(1):69. doi:10.1186/s12966-018-0691-3

Page $11 / 18$ 
19. Chaput J-P, Carson V, Gray C, Tremblay M. Importance of all movement behaviors in a 24 hour period for overall health. Int J Environ Res Public Health. 2014;11(12):12575-12581. doi:10.3390/ijerph111212575

20. Mekary RA, Lucas M, Pan A, et al. Isotemporal substitution analysis for physical activity, television watching, and risk of depression. Am J Epidemiol. 2013;178(3):474-483. doi:10.1093/aje/kws590

21. Rethorst $C D$, Moncrieft $A E$, Gellman MD, et al. Isotemporal analysis of the association of objectively measured physical activity with depressive symptoms: Results From Hispanic Community Health Study/Study of Latinos (HCHS/SOL). J Phys Act Health. 2017;14(9):733-739. doi:10.1123/jpah.2016-0648

22. Hallgren M, Nguyen T-T-D, Owen N, et al. Cross-sectional and prospective relationships of passive and mentally active sedentary behaviours and physical activity with depression. Br J Psychiatry. Published online March 21, 2019:1-7. doi:10.1192/bjp.2019.60

23. Leatherdale ST, Brown KS, Carson V, et al. The COMPASS study: A longitudinal hierarchical research platform for evaluating natural experiments related to changes in school-level programs, policies and built environment resources. BMC Public Health. 2014;14(1):331. doi:10.1186/1471-2458-14-331

24. Wong S, Leatherdale S, Manske S. Reliability and validity of a school-based physical activity questionnaire. Med Sci Sports Exerc. 2006;38:1593-1600. doi:10.1249/01.mss.0000227539.58916.35

25. Leatherdale ST, Laxer RE, Faulkner G. Reliability and Validity of the Physical Activity and Sedentary Behaviour Measures in the COMPASS Study. University of Waterloo; 2014.

26. Andresen EM, Malmgren JA, Carter WB, Patrick DL. Screening for depression in well older adults: Evaluation of a Short Form of the CES-D. Am J Prev Med. 1994;10(2):77-84. doi:10.1016/S0749-3797(18)30622-6

27. Spitzer RL, Kroenke K, Williams JBW, Löwe B. A brief measure for assessing Generalized Anxiety Disorder: The GAD7. Arch Intern Med. 2006;166(10):1092-1097. doi:10.1001/archinte.166.10.1092

28. Diener E, Wirtz D, Tov W, et al. New well-being measures: Short scales to assess flourishing and positive and negative feelings. Soc Indic Res. 2010;97(2):143-156. doi:10.1007/s11205-009-9493-y

29. Cronbach LJ. Coefficient alpha and the internal structure of tests. Psychometrika. 1951;16(3):297-334. doi:10.1007/BF02310555

30. Silva AJ, Caetano A. Validation of the Flourishing Scale and Scale of Positive and Negative Experience in Portugal. Soc Indic Res. 2013;110(2):469-478. doi:10.1007/s11205-011-9938-y

31. Growth reference 5-19 years. Accessed October 1, 2020. https://www.who.int/growthref/who2007_bmi_for_age/en/

32. Mekary RA, Willett WC, Hu FB, Ding EL. Isotemporal substitution paradigm for physical activity epidemiology and weight change. Am J Epidemiol. 2009;170(4):519-527. doi:10.1093/aje/kwp163

33. Tremblay MS, Carson V, Chaput J-P, et al. Canadian 24-hour movement guidelines for children and youth: An integration of physical activity, sedentary behaviour, and sleep. Appl Physiol Nutr Metab. 2016;41(6 (Suppl.

3)):S311-S327. doi:10.1139/apnm-2016-0151

34. Mônico-Neto M, Antunes HKM, Dattilo M, et al. Resistance exercise: A non-pharmacological strategy to minimize or reverse sleep deprivation-induced muscle atrophy. Med Hypotheses. 2013;80(6):701-705.

doi:10.1016/j.mehy.2013.02.013

35. Doré I, O'Loughlin JL, Beauchamp G, Martineau M, Fournier L. Volume and social context of physical activity in association with mental health, anxiety and depression among youth. Prev Med. 2016;91:344-350.

doi:10.1016/j.ypmed.2016.09.006

36. Huta V, Ryan RM. Pursuing Pleasure or Virtue: The Differential and Overlapping Well-Being Benefits of Hedonic and Eudaimonic Motives. J Happiness Stud. 2010;11(6):735-762. doi:10.1007/s10902-009-9171-4

37. Mack DE, Wilson PM, Gunnell KE, Gilchrist JD, Kowalski KC, Crocker PRE. Health-enhancing physical activity: Associations with markers of well-being. Appl Psychol Health Well-Being. 2012;4(2):127-150. doi:10.1111/j.1758-

Page $12 / 18$ 
0854.2012.01065.x

38. Ryff CD, Singer BH. Know thyself and become what you are: A eudaimonic approach to psychological well-being. $J$ Happiness Stud. 2008;9(1):13-39. doi:10.1007/s10902-006-9019-0

39. Steger MF, Kashdan TB, Oishi S. Being good by doing good: Daily eudaimonic activity and well-being. J Res Personal. 2008;42(1):22-42. doi:10.1016/j.jrp.2007.03.004

40. Hong E, Mason E, Peng Y, Lee N. Effects of homework motivation and worry anxiety on homework achievement in mathematics and English. Educ Res Eval. 2015;21(7-8):491-514. doi:10.1080/13803611.2015.1131721

41. Fuligni AJ, Hardway C. Daily variation in adolescents' sleep, activities, and psychological well-being. J Res Adolesc. 2006;16(3):353-378. doi:10.1111/j.1532-7795.2006.00498.x

42. Patte KA, Qian W, Leatherdale ST. Modifiable predictors of insufficient sleep durations: A longitudinal analysis of youth in the COMPASS study. Prev Med. 2018;106:164-170. doi:10.1016/j.ypmed.2017.10.035

43. Baum KT, Desai A, Field J, Miller LE, Rausch J, Beebe DW. Sleep restriction worsens mood and emotion regulation in adolescents. J Child Psychol Psychiatry. 2014;55(2):180-190. doi:10.1111/jcpp.12125

44. Talbot LS, McGlinchey EL, Kaplan KA, Dahl RE, Allison G. Sleep deprivation in adolescents and adults: Changes in affect. Emotion. 2010;10:831-841. doi:https://doi.org/10.1037/a0020138

45. Dahl RE. The regulation of sleep and arousal. Dev Psychopathol. 1996;8(1):3-27. doi:10.1017/S0954579400006945

46. Deci EL, Ryan RM. The "what" and "why" of goal pursuits: Human needs and the self-determination of behavior. Psychol Inq. 2000;11(4):227-268. doi:10.1207/S15327965PLI1104_01

47. Buxton OM, Chang A-M, Spilsbury JC, Bos T, Emsellem H, Knutson KL. Sleep in the modern family: Protective family routines for child and adolescent sleep. Sleep Health. 2015;1(1):15-27. doi:10.1016/j.sleh.2014.12.002

48. Minges KE, Redeker NS. Delayed school start times and adolescent sleep: A systematic review of the experimental evidence. Sleep Med Rev. 2016;28:86-95. doi:10.1016/j.smrv.2015.06.002

49. Patte KA, Qian W, Cole AG, et al. School start time changes in the COMPASS study: Associations with youth sleep duration, physical activity, and screen time. Sleep Med. 2019;56:16-22. doi:10.1016/j.sleep.2018.09.020

50. Dyrstad SM, Hansen BH, Holme IM, Anderssen SA. Comparison of self-reported versus accelerometer-measured physical activity. Med Sci Sports Exerc. 2014;46(1):99-106. doi:10.1249/MSS.0b013e3182a0595f

51. Araujo T, Wonneberger A, Neijens P, de Vreese C. How much time do you spend online? Understanding and improving the accuracy of self-reported measures of internet use. Commun Methods Meas. 2017;11(3):173-190.

doi:10.1080/19312458.2017.1317337

52. Smith A, Anderson M. Social media use in 2018. Published online 2018.

53. Janssen I. Estimating whether replacing time in active outdoor play and sedentary video games With active video games influences youth's mental health. J Adolesc Health. 2016;59(5):517-522.

doi:10.1016/j.jadohealth.2016.07.007

54. Reeves B, Robinson T, Ram N. Time for the human screenome project. Nature. 2020;577(7790):314-317. doi:10.1038/d41586-020-00032-5

55. Teychenne M, White RL, Richards J, Schuch FB, Rosenbaum S, Bennie JA. Do we need physical activity guidelines for mental health: What does the evidence tell us? Ment Health Phys Act. 2020;18:100315. doi:10.1016/j.mhpa.2019.100315

56. Malla A, Shah J, lyer S, et al. Youth mental health should be a top priority for health care in Canada. Can J Psychiatry. 2018;63(4):216-222. doi:10.1177/0706743718758968

57. Hoare E, Milton K, Foster C, Allender S. The associations between sedentary behaviour and mental health among adolescents: A systematic review. Int J Behav Nutr Phys Act. 2016;13(1):108. doi:10.1186/s12966-016-0432-4

Page 13/18 


\section{Tables}

Table 1

Sample Descriptives.

Page 14/18 


\begin{tabular}{|c|c|c|}
\hline Covariates & $n$ & $\%$ \\
\hline \multicolumn{3}{|l|}{ Sex } \\
\hline Male & 22514 & $48.5 \%$ \\
\hline Female & 23899 & $51.5 \%$ \\
\hline \multicolumn{3}{|l|}{ Province } \\
\hline Alberta & 2244 & $5 \%$ \\
\hline British Columbia & 7046 & $17 \%$ \\
\hline Ontario & 20311 & $49 \%$ \\
\hline Quebec & 12078 & $29 \%$ \\
\hline \multicolumn{3}{|l|}{ Grade } \\
\hline 9 & 11377 & $27 \%$ \\
\hline 10 & 12019 & $29 \%$ \\
\hline 11 & 11641 & $28 \%$ \\
\hline 12 & 6642 & $16 \%$ \\
\hline \multicolumn{3}{|l|}{ Ethnicity } \\
\hline White & 31124 & $75 \%$ \\
\hline Black & 1463 & $4 \%$ \\
\hline Asian & 5525 & $13 \%$ \\
\hline Latin American/Hispanic & 1111 & $3 \%$ \\
\hline Other/Mixed & 2456 & $6 \%$ \\
\hline \multicolumn{3}{|l|}{ Weekly Spending Money } \\
\hline Zero & 6298 & $15 \%$ \\
\hline$\$ 1-\$ 5$ & 1941 & $5 \%$ \\
\hline$\$ 6-\$ 10$ & 2479 & $6 \%$ \\
\hline$\$ 11-\$ 20$ & 4741 & $11 \%$ \\
\hline$\$ 21-\$ 40$ & 4672 & $11 \%$ \\
\hline$\$ 41-\$ 100$ & 5749 & $14 \%$ \\
\hline More than $\$ 100$ & 9469 & $23 \%$ \\
\hline Don't Know & 6330 & $15 \%$ \\
\hline \multicolumn{3}{|l|}{ BMI Classification } \\
\hline Underweight & 695 & $2 \%$ \\
\hline Normal weight & 24392 & $59 \%$ \\
\hline Overweight & 5311 & $13 \%$ \\
\hline
\end{tabular}

Page 15/18 


\begin{tabular}{|lll|}
\hline Unknown & 2434 & $6 \%$ \\
\hline Time Use and Mental Health Variables & 8847 & $21 \%$ \\
\hline Flourishing & $M$ & $S D$ \\
\hline Anxiety Symptoms & 32.0 & 5.5 \\
\hline Depression Symptoms & 6.4 & 5.5 \\
\hline Watching/Streaming TV Shows or Movies (min) & 103.5 & 76.2 \\
\hline Playing Video/Computer Games (min) & 70.5 & 95.7 \\
\hline Surfing the Internet (min) & 102.2 & 89.8 \\
\hline Texting, Messaging, Emailing (min) & 81.6 & 85.0 \\
\hline Homework (min) & 88.6 & 70.8 \\
\hline Sleep (min) & 437.3 & 79.0 \\
\hline MVPA (min) & 101.3 & 69.5 \\
\hline
\end{tabular}

Note. $N=41,679 \cdot M=$ mean $. S D=$ standard deviation .

Table 2

Isotemporal substitution model stratified by sleep. 


\begin{tabular}{|c|c|c|c|c|c|c|c|c|}
\hline & \multicolumn{4}{|c|}{$<8$ hours sleep ( $56 \%$ of sample) } & \multicolumn{4}{|c|}{${ }^{3} 8$ hours sleep ( $44 \%$ of sample) } \\
\hline & \multicolumn{4}{|c|}{ Replacement Activity } & \multicolumn{4}{|c|}{ Replacement Activity } \\
\hline & $\begin{array}{l}\text { Screen } \\
\text { time }\end{array}$ & Homework & MVPA & Sleep & $\begin{array}{l}\text { Screen } \\
\text { time }\end{array}$ & Homework & MVPA & Sleep \\
\hline \multirow[t]{2}{*}{ Anxiety } & Estimate & Estimate & Estimate & Estimate & Estimate & Estimate & Estimate & Estimate \\
\hline & {$[95 \% \mathrm{Cl}]$} & {$[95 \% \mathrm{Cl}]$} & {$[95 \% \mathrm{Cl}]$} & {$[95 \% \mathrm{Cl}]$} & {$[95 \% \mathrm{Cl}]$} & {$[95 \% \mathrm{Cl}]$} & {$[95 \% \mathrm{Cl}]$} & {$[95 \% \mathrm{Cl}]$} \\
\hline \multirow{2}{*}{$\begin{array}{l}\text { Screen } \\
\text { Time }\end{array}$} & Dropped & 0.05 & 0.00 & -0.03 & Dropped & 0.04 & -0.03 & -0.01 \\
\hline & & $\begin{array}{l}{[0.04 .} \\
0.06]\end{array}$ & $\begin{array}{l}{[-0.01} \\
0.01]\end{array}$ & $\begin{array}{l}{[-0.04,} \\
-0.02]\end{array}$ & & $\begin{array}{l}{[0.02,} \\
0.05]\end{array}$ & $\begin{array}{l}{[-0.04} \\
-0.01]\end{array}$ & $\begin{array}{l}{[-0.03,} \\
0.01]\end{array}$ \\
\hline \multirow[t]{2}{*}{ Homework } & -0.05 & Dropped & -0.05 & -0.09 & -0.04 & Dropped & -0.06 & -0.05 \\
\hline & $\begin{array}{l}{[-0.06} \\
-0.04]\end{array}$ & & $\begin{array}{l}{[-0.07} \\
-0.04]\end{array}$ & $\begin{array}{l}{[-0.10} \\
-0.07]\end{array}$ & $\begin{array}{l}{[-0.05} \\
-0.02]\end{array}$ & & $\begin{array}{l}{[-0.08} \\
-0.05]\end{array}$ & $\begin{array}{l}{[-0.07,} \\
-0.02]\end{array}$ \\
\hline \multirow[t]{2}{*}{ MVPA } & 0.00 & 0.05 & Dropped & -0.04 & 0.03 & 0.06 & Dropped & 0.02 \\
\hline & $\begin{array}{l}{[-0.01} \\
0.01]\end{array}$ & $\begin{array}{l}{[0.04} \\
0.07]\end{array}$ & & $\begin{array}{l}{[-0.05} \\
-0.02]\end{array}$ & $\begin{array}{l}{[0.01} \\
0.04]\end{array}$ & $\begin{array}{l}{[0.05,} \\
0.08]\end{array}$ & & $\begin{array}{l}{[-0.01,} \\
0.04]^{\prime}\end{array}$ \\
\hline \multirow[t]{2}{*}{ Sleep } & 0.03 & 0.09 & 0.04 & Dropped & 0.01 & 0.05 & -0.02 & Dropped \\
\hline & $\begin{array}{l}{[0.02,} \\
0.04]\end{array}$ & $\begin{array}{l}{[0.07} \\
0.10]\end{array}$ & $\begin{array}{l}{[0.02,} \\
0.05]\end{array}$ & & $\begin{array}{l}{[0.01} \\
0.02]\end{array}$ & $\begin{array}{l}{[0.04,} \\
0.06]\end{array}$ & $\begin{array}{l}{[-0.04,} \\
0.01]\end{array}$ & \\
\hline \multirow{2}{*}{$\begin{array}{l}\text { Partition } \\
\text { Model }\end{array}$} & 0.01 & 0.07 & 0.02 & -0.02 & 0.01 & 0.05 & -0.01 & 0.00 \\
\hline & $\begin{array}{l}{[0.01,} \\
0.02]\end{array}$ & $\begin{array}{l}{[0.06,} \\
0.08]\end{array}$ & $\begin{array}{l}{[0.01,} \\
0.03]\end{array}$ & $\begin{array}{l}{[-0.03} \\
-0.01]\end{array}$ & $\begin{array}{l}{[0.01} \\
0.02]\end{array}$ & $\begin{array}{l}{[0.04,} \\
0.06]\end{array}$ & $\begin{array}{l}{[-0.02} \\
0.00]\end{array}$ & $\begin{array}{l}{[-0.02,} \\
0.03]\end{array}$ \\
\hline \multirow[t]{2}{*}{ Depression } & Estimate & Estimate & Estimate & Estimate & Estimate & Estimate & Estimate & Estimate \\
\hline & [95\% Cl] & {$[95 \% \mathrm{Cl}]$} & {$[95 \% \mathrm{Cl}]$} & {$[95 \% \mathrm{Cl}]$} & {$[95 \% \mathrm{Cl}]$} & {$[95 \% \mathrm{Cl}]$} & {$[95 \% \mathrm{Cl}]$} & {$[95 \% \mathrm{Cl}]$} \\
\hline \multirow{2}{*}{$\begin{array}{l}\text { Screen } \\
\text { Time }\end{array}$} & Dropped & -0.07 & -0.04 & -0.16 & Dropped & -0.08 & -0.03 & -0.03 \\
\hline & & $\begin{array}{l}{[-0.08,} \\
-0.06]\end{array}$ & $\begin{array}{l}{[-0.05,} \\
-0.03]\end{array}$ & $\begin{array}{l}{[-0.18} \\
-0.15]\end{array}$ & & $\begin{array}{l}{[-0.09} \\
-0.07]\end{array}$ & $\begin{array}{l}{[-0.05,} \\
-0.02]\end{array}$ & $\begin{array}{l}{[-0.06,} \\
-0.01]\end{array}$ \\
\hline \multirow[t]{2}{*}{ Homework } & 0.07 & Dropped & 0.03 & -0.09 & 0.08 & Dropped & 0.05 & 0.05 \\
\hline & $\begin{array}{l}{[0.06} \\
0.08]\end{array}$ & & $\begin{array}{l}{[0.02,} \\
0.05]\end{array}$ & $\begin{array}{l}{[-0.11,} \\
-0.08]\end{array}$ & $\begin{array}{l}{[0.07,} \\
0.09]\end{array}$ & & $\begin{array}{l}{[0.03,} \\
0.06]\end{array}$ & $\begin{array}{l}{[0.02,} \\
0.07]\end{array}$ \\
\hline \multirow[t]{2}{*}{ MVPA } & 0.04 & -0.03 & Dropped & -0.12 & 0.03 & -0.05 & Dropped & 0.00 \\
\hline & $\begin{array}{l}{[0.03} \\
0.05]\end{array}$ & $\begin{array}{l}{[-0.05} \\
-0.02]\end{array}$ & & $\begin{array}{l}{[-0.14} \\
-0.11]\end{array}$ & $\begin{array}{l}{[0.02,} \\
0.05]\end{array}$ & $\begin{array}{l}{[-0.06} \\
-0.03]\end{array}$ & & $\begin{array}{l}{[-0.03,} \\
0.03]\end{array}$ \\
\hline \multirow[t]{2}{*}{ Sleep } & 0.16 & 0.09 & 0.12 & Dropped & 0.03 & -0.05 & 0.00 & Dropped \\
\hline & $\begin{array}{l}{[0.15} \\
0.18]\end{array}$ & $\begin{array}{l}{[0.08} \\
0.11]\end{array}$ & $\begin{array}{l}{[0.11,} \\
0.14]\end{array}$ & & $\begin{array}{l}{[0.01,} \\
0.06]\end{array}$ & $\begin{array}{l}{[-0.07} \\
-0.02]\end{array}$ & $\begin{array}{l}{[-0.03} \\
0.03]\end{array}$ & \\
\hline \multirow{2}{*}{$\begin{array}{l}\text { Partition } \\
\text { Model }\end{array}$} & 0.01 & -0.06 & -0.03 & -0.15 & 0.03 & -0.05 & 0.00 & 0.00 \\
\hline & $\begin{array}{l}{[0.01,} \\
0.02]\end{array}$ & $\begin{array}{l}{[-0.07} \\
-0.05]\end{array}$ & $\begin{array}{l}{[-0.04} \\
-0.01]\end{array}$ & $\begin{array}{l}{[-0.16} \\
-0.14]\end{array}$ & $\begin{array}{l}{[0.03,} \\
0.04]\end{array}$ & $\begin{array}{l}{[-0.06} \\
-0.03]\end{array}$ & $\begin{array}{l}{[-0.01} \\
0.01]\end{array}$ & $\begin{array}{l}{[-0.03,} \\
0.02]\end{array}$ \\
\hline Flourishing & Estimate & Estimate & Estimate & Estimate & Estimate & Estimate & Estimate & Estimate \\
\hline
\end{tabular}




\begin{tabular}{|lllllllll|} 
& {$[95 \% \mathrm{Cl}]$} & {$[95 \% \mathrm{Cl}]$} & {$[95 \% \mathrm{Cl}]$} & {$[95 \% \mathrm{Cl}]$} & {$[95 \% \mathrm{Cl}]$} & {$[95 \% \mathrm{Cl}]$} & {$[95 \% \mathrm{Cl}]$} & {$[95 \% \mathrm{Cl}]$} \\
\hline Screen & Dropped & 0.13 & 0.16 & 0.08 & Dropped & 0.14 & 0.16 & 0.04 \\
& & {$[0.12$,} & {$[0.15$,} & {$[0.07$,} & & {$[0.12$,} & {$[0.15$,} & {$[0.01$,} \\
& & $0.15]$ & $0.17]$ & $0.10]$ & & $0.15]$ & $0.18]$ & $0.07]$ \\
\hline Homework & -0.13 & Dropped & 0.03 & -0.05 & -0.14 & Dropped & 0.03 & -0.09 \\
& {$[-0.15$,} & & {$[0.01$,} & {$[-0.07$,} & {$[-0.15$,} & & {$[0.01$,} & {$[-0.12$,} \\
& $-0.12]$ & & $0.04]$ & $-0.03]$ & $-0.12]$ & & $0.05]$ & $-0.06]$ \\
MVPA & -0.16 & -0.03 & Dropped & -0.08 & -0.16 & -0.03 & Dropped & -0.12 \\
& {$[-0.17$,} & {$[-0.04$,} & & {$[-0.09$,} & {$[-0.18$,} & {$[-0.05$,} & & {$[-0.15$,} \\
& $-0.15]$ & $-0.01]$ & & $-0.06]$ & $-0.15]$ & $-0.01]$ & & $-0.09]$ \\
\hline Sleep & -0.08 & 0.05 & 0.08 & Dropped & -0.04 & 0.09 & 0.12 & Dropped \\
& {$[-0.10$,} & {$[0.03$,} & {$[0.06$,} & & {$[-0.07$,} & {$[0.06$,} & {$[0.09$,} & \\
& $-0.07]$ & $0.07]$ & $0.09]$ & & $-0.01]$ & $0.12]$ & $0.15]$ & \\
Partition & -0.01 & 0.12 & 0.15 & 0.07 & -0.02 & 0.11 & 0.14 & 0.02 \\
Model & {$[-0.02$,} & {$[0.11$,} & {$[0.13$,} & {$[0.06$,} & {$[-0.03$,} & {$[-0.10$,} & {$[0.13$,} & {$[-0.01$,} \\
& $-0.01]$ & $0.13]$ & $0.16]$ & $0.08]$ & $-0.02]$ & $0.13]$ & $0.15]$ & $0.05]$ \\
\hline
\end{tabular}

Note. $95 \% \mathrm{Cl}=95 \%$ confidence interval. Estimates are unstandardized. Isotemporal models are adjusted for sex, province, grade, ethnicity, SES, and BMI classification. Significant findings are bolded. 